\title{
A specific tripeptidyl substrate for tripeptidyl peptidase activity is effectively hydrolyzed by alanyl aminopeptidase/ aminopeptidase N/CD13 in the rat kidney
}

\author{
Masahiro Shibata ${ }^{1}$, Masato Koike ${ }^{2}$, Satoshi Kusumi ${ }^{1}$, Noboru Sato ${ }^{3}$, and \\ Yasuo Uchiyama ${ }^{4}$
}

\begin{abstract}
${ }^{1}$ Department of Morphological Sciences, Kagoshima University Graduate School of Medical and Dental Sciences, Kagoshima, Japan; Departments of ${ }^{2}$ Cell Biology and Neuroscience, and ${ }^{4}$ Cellular and Molecular Neuropathology, Juntendo University Graduate School of Medicine, Bunkyo-Ku, Tokyo, Japan; and ${ }^{3}$ Division of Gross Anatomy and Morphogenesis, Department of Regenerative and Transplant Medicine, Niigata University Graduate School of Medical and Dental Sciences, Niigata, Japan
\end{abstract}

Summary. L-Alanyl-L-alanyl-L-phenylalanine 4-methylcoumaryl-7-amide (AAF-MCA) is one of the classic substrates for use with tripeptidyl peptidases (TPP-I and TPP-II). We have previously clarified the tissue distribution of TPP-I in detail and noted that the protein expression of TPP-I is often incompatible with its enzyme activity. Herein, we describe the unknown peptidase, which could effectively hydrolyze AAF-MCA, in the rat kidney. The peptidase was purified after four chromatography steps, and its enzyme characteristics were elucidated. The peptidase activity was inhibited by amastatin, bestatin, and o-phenanthroline and was also inhibited by zinc and copper ions. The substrate specificity for several monoamino acidic-MCAs revealed that the peptidase had an affinity for alanyl-MCA. The

Received September 1, 2015

Address for correspondence: Masahiro Shibata, Department of Morphological Sciences, Kagoshima University Graduate School of Medical and Dental Sciences, 8-35-1, Sakuragaoka, Kagoshima 890-8544, Japan

Tel: +81-99-275-5212, Fax: +81-99-275-5214

E-mail: mshiba@m2.kufm.kagoshima-u.ac.jp amino terminal amino acid sequence of the peptidase was x-Ala-Pro-x-Leu-Pro-Gly-Ser-Thr-Ser-Ala-Thr-x-x-Ser, where $x$ indicates undetectable amino acid residues, and the antiserum against the peptidase was immunopositive for the brush border of a renal proximal tubule and the small intestine, and the surface membrane of bile canaliculi. These results indicate that the unknown peptidase that hydrolyzed AAF-MCA is the soluble form of aminopeptidase N/CD13, and caution is required when using AAF-MCA as a substrate for tripeptidyl peptidase assays.

\section{Introduction}

Tripeptidyl peptidase I (TPP-I) is a causative gene product of ceroid lipofuscinosis neuronal $2(\ln 2)$, a recessive fatal neurological disease (Sleat et al., 1997; Liu et al., 1998; Vines et al., 1998). Its defect results in the lysosomal accumulation of several proteins, particularly subunit $\mathrm{c}$ of mitochondrial ATP synthase (Ezaki et al., 1999). Therefore, subunit c is considered to be one of the native substrates of TPP-I (Ezaki et al., 2000), although the precise mechanism of the lysosomal accumulation is yet to be determined. TPP-I was originally found in 1978 (Doebber et al., 1978), and it has been purified, 
identified, and characterized from various animals and other organisms (Page et al., 1993; Vines et al., 1998; Krimper and Jones, 1999; Junaid et al., 2000). Since tripeptidyl peptidases (TPPs) specifically cleave tripeptides at the amino terminal end of proteins, chromogenic or fluorogenic tripeptide substrates have been synthesized and are normally used to measure the enzyme activities of TPP (Page et al., 1993; Junaid et al., 1999, 2000, 2001; Du et al., 2001). Among several synthetic peptides, L-alanyl-L-alanyl-L-phenylalanine 4-methyl-coumaryl-7-amide (AAF-MCA) has been most frequently used as a substrate for TPPs (Du et al., 2001; Lin et al., 2001; Warburton and Bernardini, 2001; Golabek et al., 2003). In fact, this substrate is often used for the purification of TPP-I, as mentioned above.

We previously examined the precise tissue expression and proteolytic activity of TPP-I by western blotting, immunohistochemistry, and enzyme assay using the canonical method in the various tissues of mice and rats (Koike et al., 2002). Although the TPP-I protein is expressed in the brain and kidney, the enzyme activity is much higher in the kidney than in the brain, indicating the presence of possible factors that may inhibit the activity in the brain or facilitate it in the kidney. During attempts to purify the enzyme that hydrolyzes AAF-MCA from the rat kidney, we noted the presence of the unknown peptidase that hydrolyzes AAF-MCA in rat kidney.

\section{Materials and Methods}

\section{Ethics statement}

This study was carried out in strict accordance with the recommendations in the Guide for the Care and Use of Laboratory Animals of the National Institutes of Health. The protocol was approved by the Animal Use and Care Committee guidelines of Kagoshima University.

\section{Enzyme purification}

Thirty male rats at eight weeks of age were deeply anesthetized with pentobarbital and their blood was excluded by cardiac perfusion with saline. The kidneys were quickly excised from the animals and homogenized with $570 \mathrm{ml}$ of ice-cold buffer (20 mM sodium acetate, $5 \mathrm{mM}$ EDTA, $1 \mu \mathrm{g} / \mathrm{ml}$ Leupeptin, pH5.5), and then Triton X-100 (final concentration; $1 \%$ ) was added to the homogenate and stirred for $1 \mathrm{~h}$ at $4^{\circ} \mathrm{C}$. After the sample was centrifuged at 17,000 $g$ for $1 \mathrm{~h}, 600 \mathrm{ml}$ of the obtained supernatant was dialyzed against $20 \mathrm{mM}$ sodium acetate buffer ( $\mathrm{pH}$ 5.5) and further centrifuged at $15,000 \mathrm{~g}$ for $1 \mathrm{~h}$. The subsequent supernatant, a total of $615 \mathrm{ml}$, was used for the purification of the enzyme that hydrolyzes Ala-Ala-Phe-MCA. The supernatant was applied onto a SP-Sepharose (GE Healthcare, Little Chalfont, UK) column that was equilibrated with $20 \mathrm{mM}$ sodium acetate buffer, $\mathrm{pH} 5.5$, and eluted with the same buffer containing $\mathrm{NaCl}$ that consisted of a concentration gradient (0-1M). The proteolytic activity of each fraction was measured. The fractions of major active peaks ( $88 \%$ of total activity) were concentrated with ammonium sulfate and further purified with a gel filtration column (Sephacryl H200R; GE Healthcare, Little Chalfont, UK). The active fractions were pooled and were passed through a Heparin-Sepharose (GE Healthcare, Little Chalfont, UK) column equilibrated with $20 \mathrm{mM}$ sodium acetate buffer, $\mathrm{pH}$ 5.5. The pooled enzymes were dialyzed with $20 \mathrm{mM}$ phosphate buffer, $\mathrm{pH}$ 6.0, and were passed through a Hydroxyapatite column (MacroPrep Ceramic Hydroxyapatite; BioRad, CA, USA).

\section{Enzyme assay}

Enzyme activity was measured as described elsewhere (Page et al., 1993; Koike et al., 2002). Briefly, the activities were quantified in the reaction buffer $(50 \mathrm{mM}$ sodium acetate, 1 mM EDTA, inhibitor cocktail (Roche), pH 5.5), containing $20 \mu \mathrm{M}$ substrates (Ala-Ala-Phe-MCA; Peptide Institute Inc. Osaka) that were incubated at $37^{\circ} \mathrm{C}$. The fluorescent intensity was measured with a Biolumin 960 (GE Healthcare, Little Chalfont, UK) monitored at $460 \mathrm{~nm}(\mathrm{Ex}=350 \mathrm{~nm})$ at $30 \mathrm{sec}$ intervals and the arbitrary unit of the activity was determined as the alteration of the fluorescent intensity per second. For determination of substrate specificity, each of the mono amino-acid MCAs (listed in Table I) was incubated with the reaction buffer (50 $\mathrm{mM}$ Tris- $\mathrm{HCl}, \mathrm{pH} 7.5)$. To evaluate the inhibitor spectrum or metal ion preference of the peptidase, Ala-MCA was incubated with the reaction buffer $(50 \mathrm{mM}$ Tris-HCl, pH7.5) in the presence or absence of inhibitors (listed in Table 2) or metal ions (listed in Table 3), and the fluorescent intensity was measured, as mentioned above. All enzyme assays were performed in triplicate and the relative 
activities to the control were expressed.

\section{Western blotting}

A polyclonal antiserum against the purified enzyme was prepared using rabbits by subcutaneously immunizing the purified protein. Rat tissues were homogenized with a Polytron PT3100 (KINEMATICA, Switzerland) in icecold PBS with proteinase inhibitor cocktail (Nacalai Tesque, Japan), and the homogenates were centrifuged at 1,000 $g$ for 5 min to obtain postnuclear supernatants (PNSs). PNSs were further centrifuged at $15,000 \mathrm{~g}$ for $10 \mathrm{~min}$, and the resultant supernatants were recovered. The supernatants were boiled with an SDS-PAGE sample buffer and $20 \mu \mathrm{g}$ of each sample

Table 1 Substrate specificity of the purified enzyme

\begin{tabular}{|l|c|c|}
\hline \multicolumn{1}{|c|}{ Substrate } & Rat Kidney & $\begin{array}{c}\text { Human Seminal Plasma } \\
\text { (Huang } \text { et al. 1997) }\end{array}$ \\
\hline Ala-MCA & 100 & 100 \\
\hline Met-MCA & 99 & 98 \\
\hline Phe-MCA & 76 & 53 \\
\hline Leu-MCA & 70 & 64 \\
\hline Arg-MCA & 23 & 29 \\
\hline Lys-MCA & 13 & 16 \\
\hline Pyr-MCA & 0 & 0 \\
\hline Bz-Arg-MCA & 0 & - \\
\hline
\end{tabular}

The assay proceeded at $37^{\circ} \mathrm{C}$ in $50 \mathrm{mM}$ Tris- $\mathrm{HCl}$ buffer, $\mathrm{pH} 7.5$, using the substrates listed here. Results are represented as the relative activity to the control in triplicate experiments. The results from human seminal plasma are quoted from a study by Huang et al. (1997).

Table 2 Effects of inhibitors on the peptidase activity for the purified enzyme.

\begin{tabular}{|l|c|c|}
\hline & Rat Kidney & $\begin{array}{c}\text { Human Seminal Plasma } \\
\text { (Huang } \text { et al. 1997) }\end{array}$ \\
\hline Control & 100 & 100 \\
\hline EGTA & $85(5 \mathrm{mM})$ & - \\
\hline EDTA & $77(5 \mathrm{mM})$ & $58(2 \mathrm{mM})$ \\
\hline Bestatin $(100 \mu \mathrm{M})$ & 7 & 4 \\
\hline Amastatin $(100 \mu \mathrm{M})$ & 1 & 0 \\
\hline $1,10-$ Phenanthroline & $0(1 \mathrm{mM})$ & $0(200 \mu \mathrm{M})$ \\
\hline
\end{tabular}

The assay proceeded at $37^{\circ} \mathrm{C}$ in $50 \mathrm{mM}$ Tris- $\mathrm{HCl}$ buffer, $\mathrm{pH} 7.5$, using Ala-MCA as a substrate with the inhibitors indicated here. Results are represented as the relative activity to the control in triplicate experiments. The results from human seminal plasma are quoted from a study by Huang et al. (1997). 
Table 3 Effects of divalent cations on the peptidase activity of the purified enzyme.

\begin{tabular}{|l|c|c|}
\hline & Rat Kidney & $\begin{array}{c}\text { Human Seminal Plasma } \\
\text { (Huang } \text { et al. 1997) }\end{array}$ \\
\hline Control & 100 & 100 \\
\hline $\mathrm{MgCl}_{2}$ & 97 & 100 \\
\hline $\mathrm{CaCl}_{2}$ & 102 & 95 \\
\hline $\mathrm{MnCl}_{2}$ & 56 & 84 \\
\hline $\mathrm{CoCl}_{2}$ & 42 & 60 \\
\hline $\mathrm{NiCl}_{2}$ & 29 & 63 \\
\hline $\mathrm{CuCl}_{2}$ & 5 & 4 \\
\hline $\mathrm{ZnCl}_{2}$ & 1 & 4 \\
\hline
\end{tabular}

The assay proceeded at $37^{\circ} \mathrm{C}$ in $50 \mathrm{mM}$ Tris- $\mathrm{HCl}$ buffer, $\mathrm{pH} 7.5$, using Ala-MCA as a substrate with $1 \mathrm{mM}$ of the metal ions listed here. Results are represented as the relative activity to the control in triplicate experiments. The results from human seminal plasma are quoted from a study by Huang et al. (1997).

were applied onto a 7.5\% SDS-PAGE. After transferring the gel onto a PVDF membrane, immunoblotting was performed using the antiserum $(1: 1000)$ and detected by ECL.

\section{Immunohistochemistry}

Immunohistochemistry was performed as described previously (Gotow et al., 1999). Wistar rats (8 weeks of age) were perfused through the left ventricle with saline, and then with $4 \%$ paraformaldehyde buffered with $0.1 \mathrm{M}$ phosphate buffer ( $\mathrm{pH}$ 7.2) containing 4\% sucrose. After perfusion with the fixative, various tissues including the kidneys, livers, and ileums were immediately removed from the animals and further immersed in the same fixative for $2 \mathrm{hs}$. Samples processed for paraffin embedding were cut into $5 \mu \mathrm{m}$ sections with a microtome and mounted onto glass slides coated with 3-aminopropyltriethoxysilane (Matsunami Glass IND., Ltd., Osaka Japan).

The deparaffinized sections were treated with $0.3 \% \mathrm{H}_{2} \mathrm{O}_{2}$ in methanol for $30 \mathrm{~min}$ and incubated with $2 \%$ normal goat serum for $20 \mathrm{~min}$ at room temperature (RT). They were then incubated at $4{ }^{\circ} \mathrm{C}$ with the antiserum (1:200) overnight. Further incubations were performed with biotinylated goat antirabbit IgG for $1 \mathrm{~h}$, and finally, with peroxidase-conjugated streptavidin (Vectastain ABC kit, Vector Laboratories, Burlingame, CA USA) for $1 \mathrm{~h}$ at RT. Staining for peroxidase was performed using $0.0125 \%$ 3,3'-diaminobenzidine tetrahydrochloride (DAB) and $0.002 \% \mathrm{H}_{2} \mathrm{O}_{2}$ in 0.05 M Tris$\mathrm{HCl}$ buffer ( $\mathrm{pH}$ 7.6). The counterstain was carried out with hematoxylin.

\section{Results}

\section{Purification of the unknown peptidase that hydrolyzes AAF-MCA}

We attempted to purify an unknown enzyme from the kidneys of rats via four chromatography steps using Ala-Ala-PheMCA as a substrate in an enzyme assay. From SP-Sepharose chromatography, two proteolytically active peaks were found (Fig. 1A). A minor peak that was eluted earlier had $8 \%$ of the total activity and was identified as TPP-I by western blotting (data not shown). Another peak eluted later did not contain TPP-I and possessed $88 \%$ of the total activity. We then further purified a protease from major active fractions. As shown in Figure 1B, approximately $400 \mu \mathrm{g}$ of the enzyme were purified as a homogeneous protein by four chromatography steps. The molecular mass of the purified enzyme was estimated to 
be $137 \mathrm{kDa}$ on SDS-PAGE. The amino terminal amino acid sequence of the enzyme was determined to be $\mathrm{x}$-Ala-Pro- $\mathrm{x}$ Leu-Pro-Gly-Ser-Thr-Ser-Ala-Thr-x-x-Ser, where $\mathrm{x}$ indicates undetectable amino acid residues (Fig. 1C). A database search indicated that the enzyme was virtually identical to the luminal domain of rat alanyl aminopeptidase/aminopeptidase N/CD13. The undetectable amino acid residues such as threonine or serine might have been modified residues that often undergo glycosylation or phosphorylation.

\section{Characterization of the enzyme}

We then characterized the properties of the enzyme. The substrate specificity of the purified enzyme was first studied using several mono amino acid-MCAs as substrates (Table 1), since the amino terminal amino acid sequence of the enzyme strongly suggested that the purified enzyme could be an aminopeptidase. The activity was measured at $\mathrm{pH}$ 7.5. The enzyme preferentially cleaved neutral and nonpolar amino acids such as alanine, methionine, leucine, and phenylalanine at the P1 position, while the substrates with basic amino acids such as lysine and arginine at the $\mathrm{P} 1$ position were less cleaved. Pyroglutamic acid, a common amino acid derivative in which the free amino group forms lactam, and Bz-Arginine, an N-protected amino acid, were not cleaved absolutely. The pattern of the substrate preference coincided with the results of the alanyl aminopeptidase that is seen in human seminal plasma (Table 1) (Huang et al., 1997).

Second, we examined the effect of various proteinase inhibitors on the peptidase activity of the enzyme. The activities of the purified enzyme were assayed using AlaMCA as a substrate along with several inhibitors. The results are shown in Table 2. The activity was inhibited by zinc chelator o-phenanthroline, but not by chelators such as EDTA and EGTA. The activity was almost completely inhibited by amastatin and bestatin, which are typical aminopeptidase inhibitors. The results closely resembled those of human seminal plasma aminopeptidase (Huang et al., 1997).

Third, the effects of various divalent metal ions on the proteinase activity of the purified enzyme are summarized in

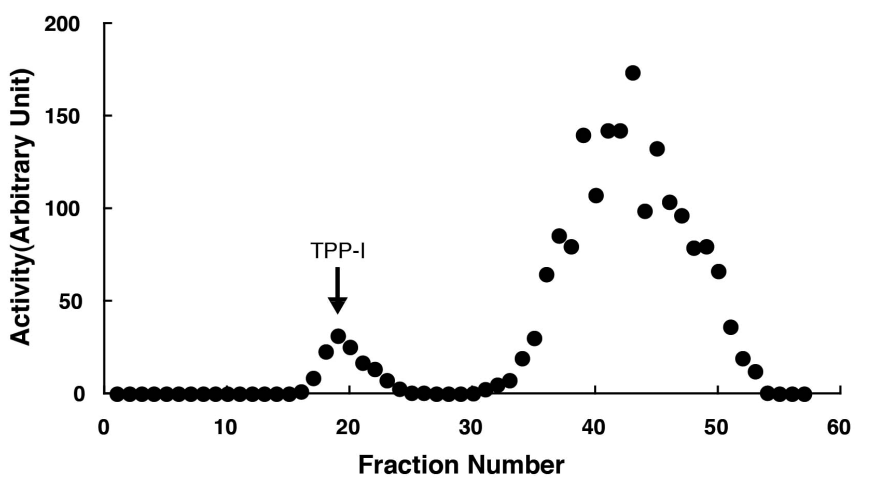

B

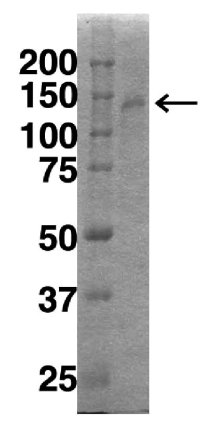

C

Human

Cytoplasm Transmembrane

Luminal/Extracellular AKGFYISKKTLGILGILLGVAAVCTIIALSVVYAQEKNRNAENSAIAPTLPGSTSATTST

Rat AKGFYISKSSLGILGILLGVAAVCTIIALSVVYSQEKNKNANSSPVASTTP-SASATT-N

Human seminal plasma STTP-SASATT-N

Rat kidney APN (this study)

Fig.1. Purification of a proteinase that hydrolyzes AAF-MCA in the kidneys of rats. A: Partially purified proteinases from crude rat kidney extracts were applied onto a SP-Sepharose column. The activities were measured using AAF-MCA as a substrate at pH 5.5, and the results were plotted. Two peaks were detected. B: SDS-PAGE was performed on a $7.5 \%$ polyacrylamide gel, and the proteins were stained with coomassie brilliant blue R-250. The arrow indicates a purified proteinase. $\mathrm{C}$ : Amino terminal sequences of aminopeptidase $\mathrm{N}$ from humans and rats are compared. A transmembrane domain is surrounded by a square. The sequence resolved in this study is placed on the last line. 
A
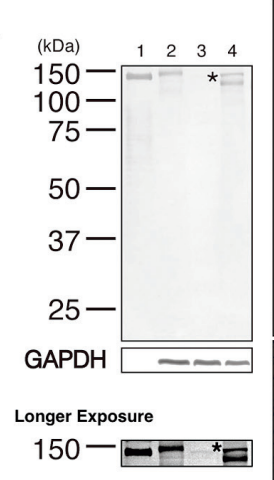
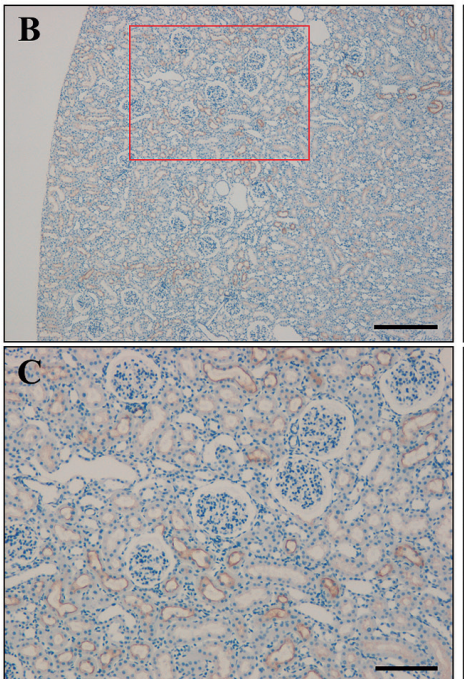
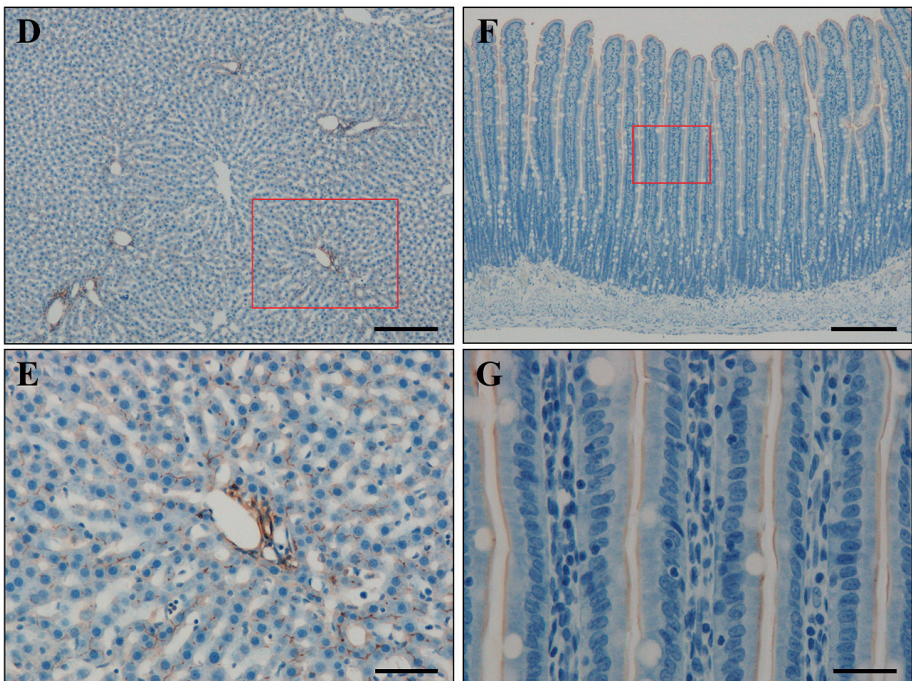

Fig. 2. The localization of the proteinase in rat tissues. A: Western blot analysis by the antiserum was carried out. A single band for the purified proteinase at a molecular mass of $137 \mathrm{kDa}$ is shown (lane 1). Twenty $\mu \mathrm{g}$ of each of the tissue lysates (lane 2; kidney, lane 3; liver, lane 4; small intestine) were applied onto a 7.5\% SDS-PAGE. A single band at a molecular mass of $140 \mathrm{kDa}$ was detected in the kidney (lane 2) and small intestine (lane 4), although a faint band was found in hepatic tissue (lane 3 in upper panel and longer exposure panel, asterisk). A smaller band at a molecular mass of $110 \mathrm{kDa}$ was also observed in the small intestine (lane 4). GAPDH was used as a loading control. BG: Immunohistochemical staining was performed in renal $(\mathbf{B}, \mathbf{C})$, hepatic $(\mathbf{D}, \mathbf{E})$, and small intestinal $(\mathbf{F}, \mathbf{G})$ tissues of rats by the antiserum. Immunosignals were detected by DAB and slides were counterstained by hematoxylin. The boxed areas in $\mathbf{B}, \mathbf{D}$, and $\mathbf{F}$ were enlarged and are shown in $\mathbf{C}, \mathbf{E}$, and $\mathbf{G}$, respectively. Positive staining is seen in the luminal surface of the renal proximal tubular epithelium (B, C), bile canaliculi $(\mathbf{D}, \mathbf{E})$, and small intestine $(\mathbf{F}, \mathbf{G})$. Bars indicate $300 \mu \mathrm{m}(\mathbf{B}), 200 \mu \mathrm{m}(\mathbf{D}, \mathbf{F}), 120 \mu \mathrm{m}(\mathbf{C}), 60 \mu \mathrm{m}(\mathbf{E})$, and $30 \mu \mathrm{m}(\mathbf{G})$.

Table 3. The activity of the enzyme was completely inhibited by zinc and copper ions, and it was strongly suppressed by nickel ions. However, the activity was inhibited by neither calcium nor magnesium ions, which have atomic radii that are larger than those of zinc, copper, or nickel ions. These results agree with those of human seminal alanyl aminopeptidase (Huang et al., 1997).

\section{Tissue distribution and localization of the protease}

To confirm that the purified enzyme was alanyl aminopeptidase, the tissue distribution and localization were analyzed using the antiserum that we generated against the purified enzyme. As shown in Figure 2, the specificity of the antiserum was confirmed by western blotting using rat tissue lysates. The purified enzyme was detected by the antiserum as a single band that showed a molecular weight of approximately 137
kDa, as shown in Figure 1 (Fig. 2A, lane1). Single protein bands immunopositive to the antiserum were detected at a molecular weight of approximately $140 \mathrm{kDa}$ in the kidney (Fig. 2A, lane2), the liver (Fig. 2A, lane3, asterisk), and in the small intestine, although the band in the liver was faint and another band appeared at a molecular mass of $110 \mathrm{kDa}$ in the small intestine. In the liver, the immunopositive band was clearly detected when an applied protein amount was appropriately increased (data not shown). The specificity of the antiserum was confirmed by western blotting.

We further examined the localization of the enzyme in rat tissues using the antiserum (Fig. 2B-G). The brush borders of the renal proximal tubular epithelial cells were immunopositive to the antiserum in the rat kidney (Fig. 2B and $\mathrm{C}$ ). No immunoreactivity to the antiserum was found in the glomeruli, in the distal renal tubular epithelial cells, or in the renal tubules in the medulla. In rat livers, positive staining for the enzyme was limited strictly to the membrane 
of the hepatocytes that consisted of bile canaliculi, but no signal could be seen in the sinusoidal wall (Fig. 2D and E). The low expression of the enzyme in the liver (Fig. 2A, lane 3 ) is thought to be due to the limited localization on the bile canaliculi. The antiserum also recognized the brush border of the epithelium in the small intestine (Fig. 2F and G). The results coincided with the localization of aminopeptidase $\mathrm{N}$ (Hersh et al., 1987).

These lines of evidence indicate that the unknown enzyme purified in this study is alanyl aminopeptidase/aminopeptidase $\mathrm{N}$.

\section{Discussion}

We have previously reported that the expression amount of TPP I protein does not reflect its enzyme activity (Koike et al., 2002). We first speculated as to the presence of unknown proteinases, or inhibitors, in various tissues that were being examined. In a previous study (Koike et al., 2002), we noticed that an unknown proteinase had hydrolyzed AAFMCA in the kidney when an increase in proteolytic activity of the enzyme was apparent. Moreover, little is known about whether mammalian cells possess inhibitors of TPP I (see MEROPS - the Peptidase Database: inhibitors for aminopeptidase N, URL <http://merops.sanger.ac.uk/cgi-bin/ inhibitors.pl?id=M01.001 >). We therefore tried to purify a peptidase that hydrolyzes AAF-MCA in the rat kidney, and found a proteinase that cleaved AAF-MCA more effectively than TPP I did in the first purification step of anion-exchange chromatography (Fig. 1A). The presence of the peptidase, alanyl aminopeptidase/aminopeptidase N/CD13, in rat kidney was confirmed based on three points: 1) a determination of the amino terminal sequence (Fig. 1C); 2) the characterization of enzymological properties (Table 1-3); and 3) the localization of the proteinase (Fig. 2B-G).

The purified aminopeptidase $\mathrm{N}$ lacked cytoplasmic and transmembrane domains as well as the aminopeptidase $\mathrm{N}$ that was purified from human seminal plasma (Huang et al., 1997). The degradation of aminopeptidase $\mathrm{N}$ by endogenous proteases must be suppressed; for this, the temperature was certainly kept at $4{ }^{\circ} \mathrm{C}$ throughout the purification process, and proteinase inhibitors were added in the crude sample. However, the electrophoretic mobility of the purified aminopeptidase $\mathrm{N}$ differed from that of the renal extract (Fig. 2A, lanes 1 and 2), and immunosignals that were indicative of aminopeptidase $\mathrm{N}$ were observed at the lumenal membrane in the renal proximal tubular epithelium, the membrane of the hepatic bile canaliculi, and the brush border of the small intestinal epithelium (Fig. 2B-G). These results suggest that the missing of the amino terminal structure could have been caused by the degradation by endogenous proteinases or autocatalysis during the course of the purification step. We detected $140 \mathrm{kDa}$ proteins in rat kidney, liver, and small intestine. Although the band that was detected in the liver was quite faint, a weak but distinct band could be observed when more than $200 \mu \mathrm{g}$ of proteins were loaded (data not shown). These $140 \mathrm{kDa}$ proteins represented the full length of aminopeptidase N (Watt and Yip, 1989). As reported by Pemberton et al. in a study using dogs, $110 \mathrm{kDa}$ proteins in the small intestine might have been a non-glycosylated form of aminopeptidase N (Pemberton et al., 1997).

We examined the AAF-MCA cleavage activity in several tissues by adding amastatin, an inhibitor of aminopeptidases (data not shown). However, the results showed that an inconsistency yet exists between TPP I protein expression levels and its levels of activity in the tissues except for the kidney. There might be other peptidase(s) which could hydrolyze AAF-MCA, or as-yet-unknown factors that suppress the activity of TPP I.

These results show that the existence of unrelated proteinase(s) should be considered when AAF-MCA and other tripeptides are used as substrates for measuring TPP I activity in crude samples. An APN inhibitor(s) is needed for assays of TPP I activity at least in renal tissues, since a major hydrolysis of AAF-MCA in the kidney is accomplished by aminopeptidase $\mathrm{N}$ (Fig. 1A).

\section{References}

Doebber TW, Divor AR, Ellis S: Identification of a tripeptidyl aminopeptidase in the anterior pituitary gland: effect on the chemical and biological properties of rat and bovine growth hormones. Endocrinology 103: 1794-1804 (1978).

Du PG, Kato S, Li YH, Maeda T, Yamane T, Yamamoto S, Fujiwara M, Yamamoto Y, Nishi K, Ohkubo I: Rat tripeptidyl peptidase I: molecular cloning, functional expression, tissue 
localization and enzymatic characterization. Biol Chem382: 1715-1725 (2001).

Ezaki J, Tanida I, Kanehagi N, Kominami E: A lysosomal proteinase, the late infantile neuronal ceroid lipofuscinosis gene (CLN2) product, is essential for degradation of a hydrophobic protein, the subunit c of ATP synthase. $J$ Neurochem 72: 2573-2582 (1999).

Ezaki J, Takeda-Ezaki M, Kominami E: Tripeptidyl peptidase I, the late infantile neuronal ceroid lipofuscinosis gene product, initiates the lysosomal degradation of subunit c of ATP synthase. J Biochem 128: 509-516 (2000).

Golabek AA, Kida E, Walus M, Wujek P, Mehta P, Wisniewski KE: Biosynthesis, glycosylation, and enzymatic processing in vivo of human tripeptidyl-peptidase I. J Biol Chem 278: 71357145 (2003).

Gotow T, Leterrier JF, Ohsawa Y, Watanabe T, Isahara K, Shibata R, Ikenaka K, Uchiyama Y: Abnormal expression of neurofilament proteins in dysmyelinating axons located in the central nervous system of jimpy mutant mice. Eur J Neurosci 11: 3893-3903 (1999)

Hersh LB, Aboukhair N, Watson S: Immunohistochemical localization of aminopeptidase $\mathrm{M}$ in rat brain and periphery: relationship of enzyme localization and enkephalin metabolism. Peptides 8: 523-532 (1987).

Huang K, Takahara S, Kinouchi T, Takeyama M, Ishida T, Ueyama H, Nishi K, Ohkubo I: Alanyl aminopeptidase from human seminal plasma: purification, characterization, and immunohistochemical localization in the male genital tract. $J$ Biochem 122: 779-787 (1997).

Junaid MA, Brooks SS, Wisniewski KE, Pullarkat RK: A novel assay for lysosomal pepstatin-insensitive proteinase and its application for the diagnosis of late-infantile neuronal ceroid lipofuscinosis. Clin Chim Acta 281: 169-176 (1999).

Junaid MA, Brooks SS, Pullarkat RK: Specific substrate for CLN2 protease/tripeptidyl-peptidase I assay. Eur J Paediatr Neurol 5: 63-68 (2001).

Junaid MA, Wu G, Pullarkat RK: Purification and characterization of bovine brain lysosomal pepstatin- insensitive proteinase, the gene product deficient in the human late-infantile neuronal ceroid lipofuscinosis. $J$ Neurochem 74 : 287-294 (2000).

Koike M, Shibata M, Ohsawa Y, Kametaka S, Waguri S, Kominami E, Uchiyama Y: The expression of tripeptidyl peptidase I in various tissues of rats and mice. Arch Histol Cytol 65: 219-232 (2002).

Krimper RP, Jones TH: Purification and characterization of tripeptidyl peptidase I from Dictyostelium discoideum. Biochem Mol Biol Int 47: 1079-1088 (1999).

Liu CG, Sleat DE, Donnelly RJ, Lobel P: Structural organization and sequence of CLN2, the defective gene in classical late infantile neuronal ceroid lipofuscinosis. Genomics 50: 206-212 (1998).

Lin L, Sohar I, Lackland H, Lobel P: The human CLN2 protein/tripeptidyl-peptidase $\mathrm{I}$ is a serine protease that autoactivates at acidic pH. J Biol Chem 276: 2249-2255 (2001).

Page AE, Fuller K, Chambers TJ, Warburton MJ: Purification and characterization of a tripeptidyl peptidase I from human osteoclastomas: evidence for its role in bone resorption. Arch Biochem Biophys 306: 354-359 (1993).

Pemberton PW, Lobley RW, Sørensen SH, Batt RM: An aminopeptidase $\mathrm{N}$ deficiency in dog small intestine. Res Vet Sci 63: 133-138 (1997).

Sleat DE, Donnelly RJ, Lackland H, Liu CG, Sohar I, Pullarkat RK, Lobel P: Association of mutations in a lysosomal protein with classical late-infantile neuronal ceroid lipofuscinosis. Science 277: 1802-1805 (1997).

Vines D, Warburton MJ: Purification and characterisation of a tripeptidyl aminopeptidase I from rat spleen. Biochim Biophys Acta 1384: 233-242 (1998).

Warburton MJ, Bernardini F The specificity of lysosomal tripeptidyl peptidase-I determined by its action on angiotensinII analogues. FEBS Lett 500: 145-148 (2001).

Watt VM, Yip CC: Amino acid sequence deduced from a rat kidney cDNA suggests it encodes the Zn-peptidase aminopeptidase N. J Biol Chem 264: 5480-5487 (1989). 